Results 26/39 (67\%) patients had at least a partial response, see Table. There was strongest concordance of Results between FeNO and LCI (70\%). 11/39 (28\%) of patients had a response in at least two domains, $4 / 39$ (10\%) at least three, and 1 patient responded in all four domains.

Conclusions In this cohort, LCI, FeNO and $\mathrm{FEV}_{1}$ were equally likely to be abnormal at baseline. FeNO and LCI were most likely to respond, (36\% and 33\% respectively), whereas $\mathrm{FEV}_{1}$ was least responsive to systemic steroids. Using this multidomain approach $67 \%$ improved over 4 weeks following treatment with systemic corticosteroid. The clinical significance of an LCI response remains to be determined. We speculate that this group may reflect a distal airway disease phenotype who may benefit from fine particle inhaled corticosteroids.

\begin{tabular}{lll}
$\begin{array}{l}\text { Abstract S70 Table } 1 \\
\text { the } 4 \text { domains tested pre- }\end{array}$ \\
Pnjection \\
$\begin{array}{lll}\text { Measurement } & \begin{array}{l}\text { Visit 1 } \\
\text { Abnormal }\end{array} & \text { Visit 2 } \\
& \text { Abnormal }\end{array}$ \\
\hline FEV 1 & $23(59 \%)$ & $19(49 \%)$ \\
LCl & $20(51 \%)$ & $7(18 \%)$ \\
ACT & $37(95 \%)$ & $26(67 \%)$ \\
FeNO & $24(62 \%)$ & $10(26 \%)$ \\
\hline
\end{tabular}

\section{S71 ARE ETHNIC DIFFERENCES IN LUNG FUNCTION EXPLAINED BY DIFFERENCES IN RESPIRATORY MUSCLE STRENGTH IN CHILDREN?}

NTS Gharbawi, G Duncan, EA Gaillard, M Viskaduraki, CS Beardsmore. University of Leicester, Leicester, UK

\subsection{6/thoraxjnl-2017-210983.77}

Background South Asian (SA) children have a reduction in forced vital capacity (FVC) of 9\%-13\% compare to white children. Ethnic differences in Maximal Inspiratory Pressure (MIP) and Maximal Expiratory Pressure (MEP) could potentially explain this. One study in adults measured MIP (but not MEP) in four ethnic groups (not including South Asians) but failed to find any differences (Sachs, Enright et al. 2009).

Aim To investigate differences in spirometry and respiratory muscle strength between white and south Asian children.

Methods Children were recruited from primary schools. We measured height, weight, and spirometry. $\mathrm{FEV}_{1}$ and FVC were expressed as Z-scores, based on predicted values for white children (Quanjer et al. 2012). For respiratory muscle strength measurements, the child breathed through a pneumotachograph attached to a shutter. To measure MIP, after several quiet breaths, the child exhaled maximally and the shutter was activated. The child made an inspiratory effort and peak pressure was recorded. The test was repeated several times. Measurements of MEP were similar, except that the child inhaled maximally and then made a forceful expiratory effort. Results We studied 263 healthy children aged 5-11 year. We obtained valid spirometry on 229 (64 white, 165 SA); valid MIP on 203 (55 white, $148 \mathrm{SA}$ ); and valid MEP on 231 (64 white, $167 \mathrm{SA}) . \mathrm{FEV}_{1}$ and $\mathrm{FVC}$ were smaller in SA children than their white peers. There were no significant differences between unadjusted MIP and MEP in white and SA children.
This finding was unchanged after adjustment for age, height and weight (Table).

Conclusions Differences in spirometry were in accordance with previous reports. We did not find any significant differences in respiratory muscle strength between the two ethnic groups. The greater FVC in white children might have been attributable to increased inspiratory muscle strength, leading to a greater volume at the start of the manoeuvre, but this was not the case. An increase in expiratory muscle strength would be less likely to increase FVC, since the end of expiration occurs when there is airway closure. Elastic recoil might be an alternative explanation for ethnic differences in lung function.

\begin{tabular}{llll}
\hline Abstract S71 Table 1 & & & \\
\hline & White & $\begin{array}{l}\text { South } \\
\text { Asian }\end{array}$ & p \\
\hline FEV 1 Z-score & 0.13 & -0.58 & $<0.000$ \\
FVC Z-score & $(1.02)$ & $(0.96)$ & \\
& 0.34 & -0.69 & $<0.000$ \\
MIP (unadjusted) (kPa) & $(1.00)$ & $(0.95)$ & \\
& 7.31 & $7.10(2.03)$ & 0.54 \\
MIP (adjusted) (kPa) & $(2.15)$ & & \\
& 7.45 & $7.05(1.91)$ & 0.19 \\
MEP (unadjusted) & $(1.92)$ & $6.48(1.73)$ & 0.38 \\
(kPa) & 6.27 & & \\
MEP (adjusted) kPa) & $(1.56)$ & $6.46(1.56)$ & 0.59 \\
\hline Values are all Mean (SD) & 6.33 & & \\
\hline
\end{tabular}

Values are all Mean (SD)

\section{S72 CLINICAL AND PATHOLOGICAL CHARACTERISTICS OF SEVERELY ASTHMATIC CHILDREN WITH PERSISTENT AIRFLOW LIMITATION}

A Nayeem, S Saglani, A Bush, LP Silveira, C Bossley, L Fleming. Imperial College London, London, UK

\subsection{6/thoraxjnl-2017-210983.78}

Introduction Severe therapy resistant asthma (STRA) in children is heterogeneous: many have normal lung function, however there is a group with persistent airflow limitation (PAL). Little is known about PAL in children and previous studies are limited by the definitions used. We hypothesised that when PAL is classified according to stringent criteria (post bronchodilator $\mathrm{FEV}_{1} \mathrm{z}$ score $<-1.96$ after a one-month systemic steroid trial (ERM 2011,Ch 5; 51-59) this group would have distinct clinical, inflammatory and pathological characteristics compared to children without PAL.

Methods Retrospective analysis of 103 STRA children. Patients were classified as STRA if they had ongoing poor control despite high dose inhaled corticosteroids plus at least one add on therapy having been assessed as part of a systematic protocol when modifiable factors such as poor adherence were identified and corrected. All children underwent bronchoscopy, bronchoalveolar lavage (BAL) and endobronchial biopsy and received intramuscular triamcinolone. Asthma control test score (ACT); inflammation (exhaled nitric oxide (FENO), induced sputum); spirometry $\left(\mathrm{FEV}_{1}\right.$. FVC) were measured on the day of bronchoscopy and 4 weeks later. The best $\mathrm{FEV}_{1}$ in the year post triamcinolone was recorded. 
Results 26/103 (25.2\%) STRA children were classified with PAL. There were no differences in the demographic characteristics between the groups. Fewer children with PAL had a previous Paediatric Intensive Care Unit admission $(21.7 \%$ versus 47.1\%); there were no other differences in asthma control. Children with PAL had a higher number of submucosal eosinophils $(p=0.021)$ in endobronchial biopsies before triamcinolone, but there were no differences in airway luminal inflammation in BAL. However, there was a trend towards lower sputum eosinophils post, but not pre, triamcinolone in children with PAL $(0.65 \% \quad(0-17)$ versus $2.5 \% \quad(0-42.8)$, $\mathrm{p}=0.054)$. There were no differences in blood eosinophils or FENO levels. All children classified as PAL post triamcinolone continued to have reduced $\mathrm{FEV}_{1}$ (post bronchodilator $\mathrm{z}$ score <-1.96) in the following 12 months.

Conclusion PAL is relatively common in paediatric STRA even when a very stringent definition is used. Mucosal eosinophilic inflammation is associated with PAL and may represent a therapeutic target. Further work is needed to elucidate underlying mechanisms.

\section{S73 COUGH FREQUENCY AND DIURNAL PATTERNS IN CHILDREN WITH ASTHMA}

D Elghamoudi, K McGuinness, J Smith, C Murray. Division of Infection, Immunity and Respiratory Medicine, Faculty of Biology, Medicine and Health, Manchester Academic Health Sciences Centre, University of Manchester and University Hospital of South Manchester NHS Foundation Trust, Manchester, UK

\subsection{6/thoraxjnl-2017-210983.79}

Background Asthma is one of the commonest childhood diseases. Although cough is considered a key symptom of asthma, little is known about the cough patterns in asthmatic children. Previously, cough patterns in asthma have only been studied subjectively, in terms of frequency and diurnal variation, and it has been reported that asthmatics cough more at night, specifically from midnight until early morning. However, there is little objective data to confirm this. Using an objective cough monitoring system we investigated cough frequency and diurnal patterns of cough over 24 hours in asthmatic children.

Methods Children (age 2-17 years) with a diagnosis of Asthma were asked to wear the VitaloJAK cough monitor for a maximum of 24 hours on two occasions - when symptoms were stable and during an exacerbation. All 24 hour recordings were processed through compression software and coughs counted by listening to the resulting files; the 24 hour cough number were reported.

Results 26 stable asthmatic children (17 male; median age 11.9 years) completed 24 hour recordings when stable; 12 repeated recordings during an exacerbation. During the stable period median total cough counts during awake-hours, sleephours and 24 hour periods were 69.5 (range 3-395), 0 (range 0-151) and 71 (range 4-432) respectively. Coughs occurred mostly during the awake time (86\%), peaking at 08:00 and 19:00 hours, and rarely occurred during the night when subjects were sleeping (14\%). During exacerbations the median total cough counts during awake-hours, sleep-hours and 24 hours were 183 (range 0-632), 18.5 (range 0-128) and 262 (range 10-645), which were significantly higher than during stable recordings $(\mathrm{p}<0.05)$. The incidences of day-time cough were similar to that of stable asthmatic recordings $(80 \%)$ and the incidences of night-time cough remained low (10\%). However, during an exacerbation, the peak time of coughing was at 11:00 and 17:00-19:00 hours. The distribution of cough for 12 asthmatic children when stable and during exacerbations is shown in figure 1.

Conclusions Objective monitoring shows that cough frequency in children is greater during the day and was reduced during

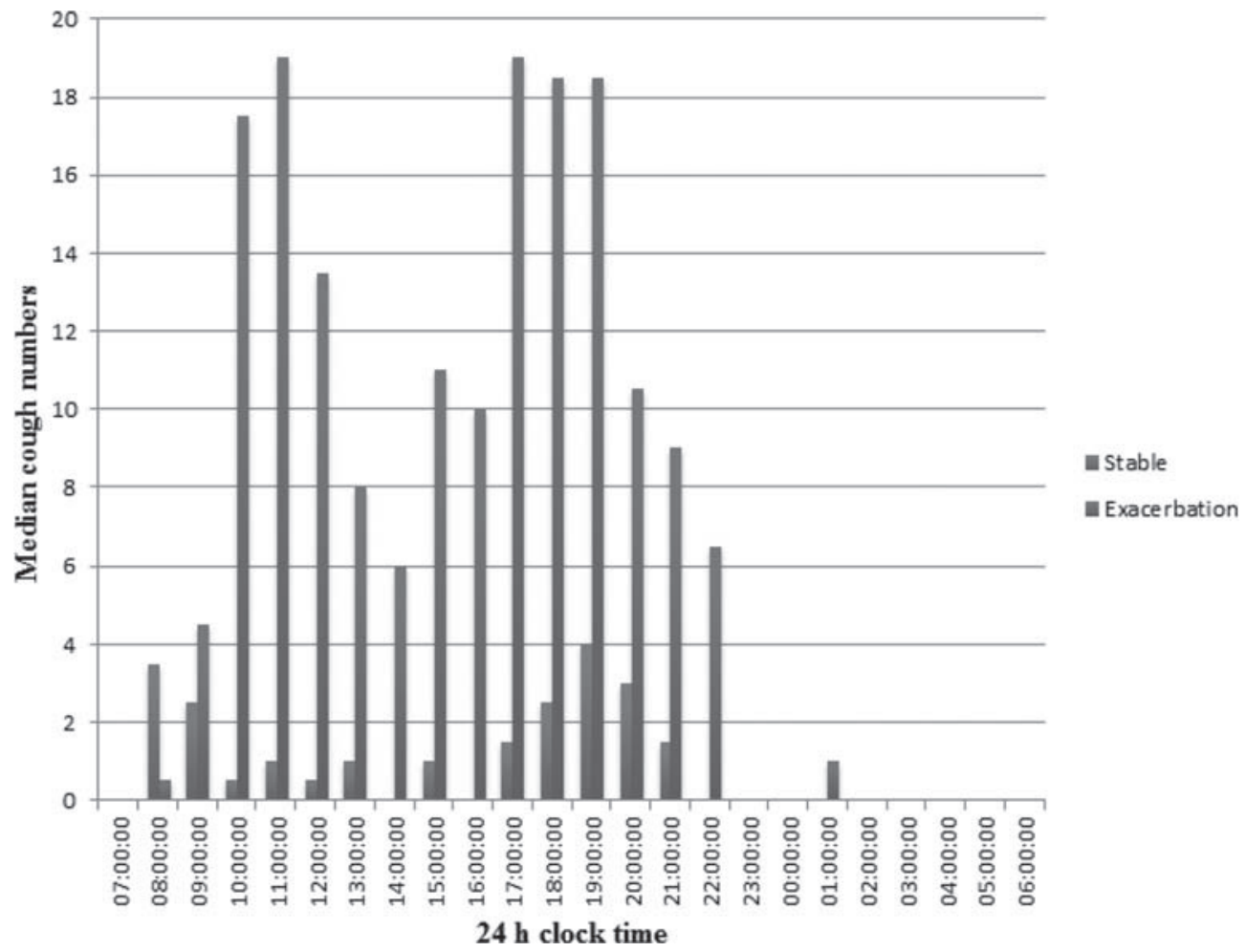

Abstract S73 Figure 1 Cough frequency distribution during exacerbations and stable asthma for 12 asthmatic children. 